\title{
Genetic Variation of Inflammatory Genes to Ischemic Stroke Risk in a Chinese Han Population
}

\author{
Zhongqiu Zhang ${ }^{1,2, *}$ \\ Yanping $\mathrm{Mei}^{3}$ ** \\ Mengqiu Xiong ${ }^{3}$ \\ Fang Lu ${ }^{1,2}$ \\ Xianghong Zhao ${ }^{1,2}$ \\ Junrong Zhu ${ }^{1,2}$ \\ Bangshun $\mathrm{He}$ (D) ${ }^{1,3}$ \\ 'School of Basic Medicine \& Clinical \\ Pharmacy, China Pharmaceutical \\ University, Nanjing, 21 I I98, Jiangsu \\ Province, People's Republic of China; \\ ${ }^{2}$ Department of Pharmacy, Nanjing First \\ Hospital, Nanjing Medical University, \\ Nanjing, 2 10006, Jiangsu Province, \\ People's Republic of China; ${ }^{3}$ Department \\ of Laboratory Medicine, Nanjing First \\ Hospital, Nanjing Medical University, \\ Nanjing, 210006, Jiangsu Province, \\ People's Republic of China \\ *These authors contributed equally to \\ this work
}

Background: Inflammation proteins play an important role in stroke occurrence. IL1A, $I L 1 B, P T G S 2, M M P 2$, and $M M P 9$ were the mediators involved in the immune response, and the association of these genetic variations with ischemic stroke (IS) risk was still unclear.

Methods: To investigate the susceptibility of genetic variations of $I L 1 A, I L 1 B, P T G S 2$, $M M P 2$, and $M M P 9$ to IS risk, we performed a case-control study involving 299 patients and 300 controls in a Chinese population. Thirteen genetic variations of investigated genes of all participants were genotyped using an improved multiplex ligase detection-reaction technique.

Results: No SNP in all genes showed an association with overall IS. However, in subgroup analysis, PTGS2 rs689466 (dominant model: CT vs TT $-\mathrm{OR}_{\text {adjusted }}=2.51,95 \%$ CI: 1.22 5.16, $p=0.012$; co-dominant model: $\mathrm{CT} / \mathrm{CC}$ vs $\mathrm{TT}-\mathrm{OR}_{\text {adjusted }}=2.53,95 \% \mathrm{CI}: 1.26-5.07$, $\mathrm{p}=0.009$; additive model $\left.-\mathrm{OR}_{\text {adjusted }}=2.26,95 \% \mathrm{CI}: 1.19-4.28, p=0.013\right)$ and $\mathrm{rs} 5275$ (dominant model: GG vs AA $-\mathrm{OR}_{\text {adjusted }}=0.31,95 \% \mathrm{CI}: 0.12-0.80, p=0.016$; co-dominant model: $\mathrm{GA} / \mathrm{GG}$ vs $\mathrm{AA}-\mathrm{OR}_{\text {adjusted }}=0.45,95 \% \mathrm{CI}: 0.21-0.95, p=0.036$; additive model $\mathrm{OR}_{\text {adjusted }}=0.60,95 \%$ CI: $0.39-0.92, p=0.020$ ) were associated with IS type of small-vessel occlusion.

Conclusion: Our study suggested that PTGS2 rs689466 C and rs5275 A were potentially associated with IS subtype of small-vessel occlusion. Our result should be confirmed with further large sample sized studies.

Keywords: ischemic stroke, genetic variation, IL1A,IL1B, PTGS2, MMP2, MMP9, risk

\section{Introduction}

Stroke is a neurological disease caused by vascular, leading to considerably high disability and mortality. The Global Burden of Diseases, Injuries, and Risk Factors Study (GBD) showed that stroke and ischemic heart were the top cause of disability over the age of $50 .{ }^{1}$ Depending on regional epidemiology, stroke is classified as ischemic stroke (IS) and hemorrhagic stroke, and the most common of which is IS, accounting for $87 \%$ of all stroke patients.

In the clinical setting, according to the etiology of ischemic, five subtypes were divided for IS, which was developed for the TOAST study: $\left.{ }^{2} 1\right)$ large-artery atherosclerosis, 2) small-vessel occlusion, 3) cardioembolism, 4) other determined etiology, and 5) undermined etiology. The most common cause of ischemic is due to arterial occlusion, and the rarer cause is cerebral veins or venous sinuses. ${ }^{3}$ There are many causes of stroke, such as hypertension, diabetes, dyslipidemia, smoking, and genetic background. Especially, genetic background accounts for $30-40 \%$ of IS. ${ }^{4}$
Correspondence: Bangshun He Department of Laboratory Medicine, Nanjing First Hospital, Nanjing Medical University, Nanjing, Jiangsu Province, Junrong Zhu

Department of Pharmacy, Nanjing First Hospital, Nanjing Medical University, 68 Changle Road, Nanjing, Jiangsu Province, 210006, People's Republic of China Email junrong_zhu@aliyun.com 
Inflammation contributes across the spectrum of IS. ${ }^{5}$ Firstly, inflammation might not only promote thrombus formation but also inhibit endogenous fibrinolysis to enhance clot stability. ${ }^{6}$ Secondly, the inflammatory mediators promote immune cells and solutes into the brain parenchyma and damage the blood-brain barrier further, and contribute the thrombogenesis. ${ }^{7,8}$ Moreover, some inflammatory mediators might be the predictor factors of IS clinical outcome. ${ }^{9,10}$ Multiple inflammation-associated mediators are involved in the process. ${ }^{11}$ For example, the interleukin (IL)-1 family, as a highly active proinflammatory cytokine, is a main regulator of inflammation and triggers inflammatory cascade response by binding to the IL1 receptor. ${ }^{12} I L 1 A$ and $I L 1 B$ are distinct members of $I L 1$ genes and encode IL $1 \alpha$ and IL1 $\beta$, respectively. Experimental studies show that abnormal IL1 $\alpha$ and IL1 $\beta$ levels will lead to inflammatory diseases ${ }^{12}$ and involve a variety of cellular activities, including adhesion molecule induction and procoagulant activity, which present a higher inflammatory reaction and affect the development of atherosclerosis resulting in IS. ${ }^{13}$ Studies showed IL1 $\beta$ had significantly higher blood levels for stroke ${ }^{14}$ and contribute to the occurrence of stroke. ${ }^{15}$ Prostaglandin-endoperoxide synthase (PTGS2), known as COX2, is an important induced enzyme in vascular endothelial cells, smooth muscle cells, and platelets. It is induced by cytokines and growth factors ${ }^{16}$ and is closely associated with the formation of atherosclerosis. ${ }^{17}$ Multiple studies reported an association between PTGS2 genetic polymorphisms and IS risk. ${ }^{18,19}$ Matrix metalloproteinases (MMPs), as a category of proteolytic zinc-dependent enzymes, play an important role in the degradation of the extracellular matrix and take part in the progression of atherosclerosis by triggering the migration and proliferation of smooth muscle cells and by causing destabilization of atherosclerotic plaques. ${ }^{20}$ Previously, studies have reported that the MMPs protein levels elevate result in blood-brain barrier dysfunction and impact the extent of the infarct, ${ }^{21-23}$ and the polymorphisms of $M M P$ s have been evaluated in IS. ${ }^{24,25}$ The association with IS of MMPs is focused on $M M P 2$ and $M M P 9^{26}$, and the high expression level of MMP2 and MMP9 has been reported that was associated with increased risk of IS and major disability. ${ }^{15,27-29}$

Given the putative role of the above gene involved in the occurrence of IS, to identify the risk of the genetic background to IS risk, we conducted a case-control association study to assess the association between 13 single nucleotide polymorphisms (SNPs) in IL1B, IL1A, PTGS2, $M M P$ 9, $M M P 2$, and risk of IS.

\section{Materials and Methods}

\section{Study Subjects}

A total of 299 patients diagnosed based on clinical symptoms, physical examination, and head computed tomography or magnetic resonance imaging, and 300 healthy controls were enrolled in this case-control study. ${ }^{30}$ All patients presented with signs and symptoms lasting more than 24 hours which sudden onset of focal or global neurological deficits. Patients with a history of transient ischemic attacks, hemorrhagic stroke, cerebral trauma, cardiogenic thrombosis, coagulation disorders, autoimmune disease, tumors, or peripheral vascular disease were excluded. The etiology of IS was classified according to the Trial of Org 10172 in Acute Stroke Treatment (TOAST) as large-artery atherosclerosis, cardioembolism, small-vessel atherosclerosis, and stroke of other etiology.

Healthy control subjects were recruited during the same period from the Health Medical Center of Nanjing First Hospital. The healthy controls were confirmed according to the routine health examination results. For the controls, the hematologic diseases, tumors, autoimmune diseases, liver ailments, and genetic diseases were excluded. All enrolled participants come from the same geographic region: Nanjing City, Jiangsu, China. The patient information was collected from the hospital information system, and the health control information was from questionnaires. Declaration of Helsinki and all procedures were approved by the Institutional Review Board of Nanjing First Hospital, and all participants were written informed consent.

\section{DNA Extraction and Genotyping}

The SNPs of IL1A, IL1B, PTGS2, MMP2, and MMP9 were selected and retrieved from the National Center for Biotechnology Information dbSNP database (https://www. ncbi.nlm.nih.gov/snp/), and then selected potential genes based on the following criteria: 1) positioned in exons, promoter regions, 5'UTRs, 3'UTRs, or splice sites; 2) minor-allele frequency $\geq 5 \%$; and 3 ) had been reported to be associated with IS risk. Finally, thirteen genetic variations were selected (see Table S1 for details). The DNA extraction and genotyping were performed as previously described. ${ }^{30}$ Genotyping used a method based on an improved multiplex ligase detection reaction technique developed by Genesky Biotech (Shanghai, China). In detail, firstly, genetic-variation loci were amplified by multiplex polymerase chain reaction, and then the amplification products were purified with nuclease and shrimp 
alkaline enzyme. Finally, each locus contained two 5'terminal allele-specific probes and a 3 'terminal-specific probe of fluorescent tags, and ligation products were analyzed with an ABI 3730XL finally.

\section{Statistical Analysis}

For the distribution of genotypes, a goodness-of-fit Chisquare test was adopted to test the Hardy-Weinberg equilibrium (HWE) in the control group. Differences in the demographic characteristics of the two groups were assessed by $t$-test or $\chi^{2}$ test. Logistic regression was applied to calculate the susceptibility of polymorphisms to IS risk with ORs and 95\% CIs based on different genetic models: Dominant model (Rare allele homozygote (RR) or heterozygous (WR) vs wild-type homozygote (WW) genotypes), co-dominant model (RR+WR vs $\mathrm{WW}$ ), and additive model (WW vs WR vs RR). ${ }^{31} \mathrm{P}<$ 0.05 was considered statistically significant.

\section{Results}

\section{Characteristics of the Study Population}

A total of 299 patients with IS and 300 healthy controls were enrolled in this study. Their demographic data and clinical characteristics are summarized in Table S2. There were no significant statistical differences in sex ( $p=0.312)$, drinking $(p=1.000)$, or chol $(p=0.623)$, but there were significant differences between the two groups with age $(p<0.001)$, smoking $(p<0.001)$, hypertension $(p<0.001)$. For clinical characteristics, levels of TG $(p<0.001)$, GLU $(p<0.001)$, HYC $(p<0.001)$, and CRP $(p<0.001)$ were significantly higher in patients than in controls. In contrast, levels of HDL in patients were significantly lower than in controls $(p=0.004)$. A total of 56 patients were identified as having small-vessel occlusion, 116 having large-artery, 28 having cardioembolism, and 99 having other etiologies. The HWE result showed that all genotypes were not derived from controls (Table S1).

\section{Association Between and Risk of Stroke}

Logistic regression analysis revealed that no SNP showed any association with the risk of IS in all genes (Table 1) and subgroup stratified by sex (Table 2). However, subgroup analysis by subtypes of IS showed that PTGS2 rs689466 (dominant model: CT vs TT $-\mathrm{OR}_{\text {adjusted }}=2.51$, 95\% CI: 1.22-5.16, $p=0.012$; co-dominant model: CT/CC vs $\mathrm{TT}-\mathrm{OR}_{\text {adjusted }}=2.53$, 95\% CI: $1.26-5.07, p=0.009$; additive model $-\mathrm{OR}_{\text {adjusted }}=2.26$, 95\% CI: $1.19-4.28$, $p=0.013$ ) was associated with increased risk of IS type of small-vessel occlusion. However, the PTGS2 rs5275 (dominant model: $\mathrm{GG}$ vs $\mathrm{AA}-\mathrm{OR}_{\text {adjusted }}=0.31,95 \% \mathrm{CI}$ : $0.12-0.80, p=0.016$; co-dominant model: $\mathrm{GA} / \mathrm{GG}$ vs $\mathrm{AA}-$ $\mathrm{OR}_{\text {adjusted }}=0.45,95 \% \mathrm{CI}: 0.21-0.95, p=0.036$; additive model $-\mathrm{OR}_{\text {adjusted }}=0.60,95 \%$ CI: $0.39-0.92, p=0.020$ ) were associated with decreased risk of IS type of smallvessel occlusion. Additionally, the SNP MMP9 rs3918242 was observed to be susceptible to the cardioembolic subtype of IS with dominant model (CT vs CC - OR $\mathrm{Odjusted}$ $=0.31,95 \%$ CI: $0.11-0.91, p=0.033)$ but not other genetic models (Table 3 ). No other genotypes were observed significant differences in stroke risk.

\section{Discussion}

The population-based case-control association study had not observed associated SNPs with overall IS. However, PTGS2 rs689466 (C allele) and rs5275 (A allele) were associated with IS subtype of small-vessel occlusion.

Inflammatory proteins play an important role in the pathogenesis of stroke. Inflammatory genes, such as C-reactive protein $(C R P)$, interleukin $(I L)$ 6, transforming growth factor $\beta 1$ (TGFB1), were identified to contribute to stroke risk. ${ }^{11,32-34}$ It is well known that the elevated levels of inflammatory markers may reflect a high burden of atherosclerosis and thrombosis, which contribute to the occurrence of IS. ${ }^{13,35}$

PTGS2, with loci on chromosome 1, is an inducible enzyme that catalyzes arachidonic acid into prostaglandins and plays a vital role in inflammation. ${ }^{36}$ The expression of $P T G S 2$ is increased significantly in stroke with inflammatory cells infiltrating, and relevant studies have reported that it is associated with IS. ${ }^{18}$ The rs689466 (PTGS2 -1195T>C) is located in the PTGS2 promoter, and the $-1195 \mathrm{~T}$ allele displays a higher PTGS2 level than the $-1195 \mathrm{C}$ allele. ${ }^{37}$ The rs5275 $(8473 \mathrm{~A}>\mathrm{G})$ is located in PTGS2 $3^{\prime}$-UTR, and the 8273 A allele could regulate $P T G S 2$ higher expression by maintaining the stability of PTGS2 mRNA, ${ }^{38,39}$ indicating rs5275A allele, associated with increased PTGS2 expression, induce the risk of IS. According to our study, rs689466 (C allele) and rs5275 (A allele) were associated with smallvessel occlusion whether dominant model or additive model, but have no significant difference in all IS. The reason may be that PTGS2 induces thrombosis ${ }^{17}$ which causes thrombotic occlusion of small vessels. ${ }^{37}$ Chen reported that rs689466 was associated with all IS and the effects were confined to small-vessel occlusion but not large-artery. ${ }^{37}$ 
Table I Genotype Distribution of the Polymorphisms in All Participants

\begin{tabular}{|c|c|c|c|c|}
\hline Genotype & Patients, $n(\%)$ & Controls, $n(\%)$ & $\operatorname{OR}(95 \% \mathrm{CI})^{\mathrm{a}}$ & $P$ value \\
\hline \multicolumn{5}{|l|}{ ILIB } \\
\hline \multicolumn{5}{|l|}{ rsl6944 } \\
\hline AA & $77(25.75)$ & $89(29.67)$ & Reference & \\
\hline GA & $\mid 55(5 \mid .84)$ & I47(49.00) & $1.10(0.74-1.65)$ & 0.641 \\
\hline GG & $67(22.4 I)$ & $64(21.33)$ & I.I I(0.68-I.82) & 0.672 \\
\hline GA/GG & $222(74.25)$ & $211(70.33)$ & $1.12(0.76-1.63)$ & $0.57 I$ \\
\hline \multicolumn{5}{|c|}{ rsII43627 } \\
\hline GG & $77(25.75)$ & $88(29.33)$ & Reference & \\
\hline GA & $|5|(50.50)$ & $145(48.33)$ & $I .06(0.7 I-I .59)$ & 0.779 \\
\hline AA & $7 I(23.75)$ & $67(22.33)$ & $1.13(0.69-1.83)$ & 0.632 \\
\hline GA/AA & $222(74.25)$ & $212(70.67)$ & $1.09(0.75-1.60)$ & 0.647 \\
\hline \multicolumn{5}{|c|}{ rsII43634 } \\
\hline GG & $285(95.32)$ & $288(96.00)$ & Reference & \\
\hline GA & $12(4.01)$ & $12(4.00)$ & $0.87(0.37-2.05)$ & 0.750 \\
\hline AA & $2(0.67)$ & $0(0.00)$ & - & 0.988 \\
\hline GA/AA & I4(4.68) & $12(4.00)$ & $0.96(0.42-2.19)$ & 0.917 \\
\hline \multicolumn{5}{|l|}{ ILIA } \\
\hline \multicolumn{5}{|c|}{ rs|800587 } \\
\hline GG & $239(79.93)$ & $252(84.00)$ & Reference & \\
\hline GA & $56(\mid 8.73)$ & $45(15.00)$ & $1.33(0.85-2.09)$ & 0.213 \\
\hline AA & $4(1.34)$ & $3(1.00)$ & $1.02(0.21-4.99)$ & 0.977 \\
\hline GA/AA & $60(20.07)$ & $48(16.00)$ & $1.31(0.84-2.03)$ & 0.230 \\
\hline \multicolumn{5}{|l|}{ PTGS2 } \\
\hline \multicolumn{5}{|l|}{ rs204I7 } \\
\hline $\mathrm{CC}$ & $265(88.63)$ & $266(88.67)$ & Reference & \\
\hline GC & $34(I 1.37)$ & $34(11.33)$ & $1.03(0.60-1.76)$ & 0.917 \\
\hline GG & $0(0)$ & $0(0)$ & - & - \\
\hline GC/GG & $34(11.37)$ & $34(11.33)$ & $1.03(0.60-1.76)$ & 0.917 \\
\hline \multicolumn{5}{|l|}{ rs689466 } \\
\hline TT & $197(65.89)$ & $180(60.00)$ & Reference & \\
\hline $\mathrm{CT}$ & $95(31.77)$ & $109(36.33)$ & $0.80(0.56-1.15)$ & 0.222 \\
\hline $\mathrm{CC}$ & $7(2.34)$ & $\mathrm{II}(3.67)$ & $0.52(0.19-1.42)$ & 0.201 \\
\hline $\mathrm{CT} / \mathrm{CC}$ & $102(34.11)$ & $120(40.00)$ & $0.77(0.54-1.10)$ & 0.149 \\
\hline \multicolumn{5}{|l|}{ rs5275 } \\
\hline $\mathrm{AA}$ & $90(30.10)$ & $98(32.67)$ & Reference & \\
\hline GA & $139(46.49)$ & $149(49.67)$ & $0.98(0.67-1.45)$ & 0.937 \\
\hline GG & $70(23.4 I)$ & $53(17.67)$ & $1.54(0.93-2.55)$ & 0.094 \\
\hline GA/GG & $209(69.9)$ & $202(67.33)$ & I.I0(0.77-I.59) & 0.597 \\
\hline \multicolumn{5}{|l|}{ MMP9 } \\
\hline \multicolumn{5}{|l|}{ rsI7576 } \\
\hline GG & $150(50.17)$ & $155(5 \mid .67)$ & Reference & \\
\hline GA & $118(39.46)$ & $114(38.00)$ & $1.04(0.72-1.49)$ & 0.843 \\
\hline AA & $31(10.37)$ & $31(10.33)$ & $1.03(0.59-1.82)$ & 0.908 \\
\hline GA/AA & $149(49.83)$ & $145(48.33)$ & $1.03(0.74-1.45)$ & 0.849 \\
\hline
\end{tabular}

(Continued) 
Table I (Continued).

\begin{tabular}{|c|c|c|c|c|}
\hline Genotype & Patients, $n(\%)$ & Controls, $n(\%)$ & $\operatorname{OR}(95 \% \mathrm{Cl})^{\mathrm{a}}$ & $P$ value \\
\hline \multicolumn{5}{|c|}{ rs3918242 } \\
\hline $\mathrm{CC}$ & $245(81.94)$ & $254(84.67)$ & Reference & \\
\hline $\mathrm{CT}$ & $51(17.06)$ & $42(14.00)$ & $1.37(0.86-2.18)$ & 0.193 \\
\hline $\mathrm{TT}$ & $3(1.00)$ & $4(1.33)$ & $0.86(0.17-4.36)$ & 0.852 \\
\hline $\mathrm{CT} / \mathrm{TT}$ & $54(18.06)$ & $46(15.33)$ & I.32(0.84-2.08) & 0.230 \\
\hline \multicolumn{5}{|l|}{ rs9509 } \\
\hline TT & $189(63.21)$ & $184(6 \mid .33)$ & Reference & \\
\hline $\mathrm{CT}$ & $100(33.44)$ & $101(33.67)$ & $0.94(0.66-1.35)$ & 0.750 \\
\hline $\mathrm{CC}$ & $10(3.34)$ & $15(5.00)$ & $0.65(0.27-1.54)$ & 0.325 \\
\hline $\mathrm{CT} / \mathrm{CC}$ & $110(36.79)$ & $116(38.67)$ & $0.91(0.64-1.28)$ & 0.575 \\
\hline \multicolumn{5}{|l|}{ MMP2 } \\
\hline \multicolumn{5}{|l|}{ rs720I } \\
\hline AA & $173(57.86)$ & I78(59.33) & Reference & \\
\hline$C A$ & $105(35.12)$ & $103(34.33)$ & $0.90(0.62-1.29)$ & 0.556 \\
\hline $\mathrm{CC}$ & $2 \mathrm{I}(7.02)$ & $19(6.33)$ & I.I3(0.56-2.29) & $0.74 I$ \\
\hline $\mathrm{CA} / \mathrm{CC}$ & $126(42.14)$ & $122(40.67)$ & $0.93(0.66-1.32)$ & 0.694 \\
\hline \multicolumn{5}{|c|}{ rs2285053 } \\
\hline $\mathrm{CC}$ & $17 \mid(57.19)$ & I76(58.67) & Reference & \\
\hline $\mathrm{CT}$ & $102(34.11)$ & $103(34.33)$ & $1.03(0.7|-| .48)$ & 0.895 \\
\hline $\mathrm{TT}$ & $26(8.70)$ & $21(7.00)$ & $1.39(0.73-2.64)$ & 0.311 \\
\hline $\mathrm{CT} / \mathrm{TT}$ & $\mid 28(42.8 \mid)$ & $\mid 24(4 \mid .33)$ & $1.08(0.77-1.53)$ & 0.643 \\
\hline \multicolumn{5}{|l|}{ rs243864 } \\
\hline TT & $238(79.60)$ & $238(79.33)$ & Reference & \\
\hline $\mathrm{CT}$ & $56(18.73)$ & $58(19.33)$ & $0.89(0.57-1.37)$ & 0.583 \\
\hline $\mathrm{CC}$ & $5(1.67)$ & $4(1.33)$ & $2.45(0.60-9.91)$ & 0.210 \\
\hline CT/CC & $6 \mathrm{I}(20.40)$ & $62(20.67)$ & $0.95(0.63-1.45)$ & 0.821 \\
\hline
\end{tabular}

Note: ${ }^{a}$ Adjusted for age, sex, smoking, and drinking.

Shan also showed that rs689466 was associated with small vessel disease, ${ }^{40}$ which was consistent with our results. However, Zhao reported that rs68944 has a higher risk in LAA was dissimilar to our study, ${ }^{41}$ but its gene-gene interactions of three PTGS genes including rs689466 were an association with small vessel occlusion. For rs5275, another PTGS2 associate gene, few studies have discussed the association between rs5275 and stroke risk to date, but it was reported that was impacted the IS outcome. ${ }^{42}$ Our study showed that rs5275 was associated with small-vessel occlusion as well as rs689466, so more large sample studies need to be conducted.

$M M P 9$ locates on chromosome $20 \mathrm{q} 12-\mathrm{q} 13$, is a member of the MMP family, and plays a role in the progression of IS. $^{43}$ The MMP9 rs3918242, within the promoter region $(-1562 \mathrm{C}>\mathrm{T}), \mathrm{T}$ allele led to a higher promoter activity of the MMP9. ${ }^{44-46}$ The conclusions of rs3918242 T allele for stroke risk were controversial. ${ }^{47-49}$ A meta-analysis including 14 control studies with 3233 IS patients and 3123 controls showed that $M M P 9$ rs3918242 variants contributed to increasing the risk of IS, ${ }^{49}$ and the report showed that T allele carriers of rs3918242 polymorphism probably contributed to increasing in IS than $\mathrm{C}$ allele. However, our study shows that $\mathrm{CT}$ decreases the risk of cardioembolic stroke than $\mathrm{CC}$. The reason for the contradiction with previous studies may be due to smaller patients included in this study in that only 7 patients with CT genes and no TT genes among the patients with cardioembolic stroke in this study, which may affect the statistical power. Moreover, there was no significant difference under the additive model. For the difference above, our result should be verified by further large sample sized study.

A total of thirteen SNPs were also included in this study. Although these SNPs have been reported to affect the expression of some inflammatory proteins that contribute to the risk 
Table 2 Genotype Distribution of Polymorphisms in All Participants Stratified by Sex

\begin{tabular}{|c|c|c|c|c|}
\hline \multirow[t]{2}{*}{ Genotype } & \multicolumn{2}{|c|}{ Male } & \multicolumn{2}{|c|}{ Female } \\
\hline & $\operatorname{OR}(95 \% \mathrm{CI})^{\mathrm{a}}$ & P-value ${ }^{a}$ & $\operatorname{OR}(95 \% \mathrm{CI})^{\mathrm{a}}$ & P-value ${ }^{a}$ \\
\hline \multicolumn{5}{|l|}{ ILIB } \\
\hline \multicolumn{5}{|l|}{ rsI6944 } \\
\hline AA & Reference & & Reference & \\
\hline GA & $1.35(0.80,2.29)$ & 0.264 & $0.75(0.37,1.52)$ & 0.424 \\
\hline GG & $1.37(0.72,2.61)$ & 0.335 & $0.88(0.38,2.07)$ & 0.759 \\
\hline GA/GG & $1.36(0.83,2.23)$ & 0.223 & $0.81(0.42,1.55)$ & 0.517 \\
\hline Additive model & I.I8(0.86,I.6I) & 0.302 & $0.90(0.59,1.39)$ & 0.636 \\
\hline \multicolumn{5}{|l|}{ rsII43627 } \\
\hline GG & Reference & & Reference & \\
\hline GA & $1.26(0.74,2.14)$ & 0.402 & $0.76(0.38,1.55)$ & 0.454 \\
\hline AA & I.44(0.77,2.70) & 0.259 & $0.83(0.36,1.94)$ & 0.671 \\
\hline GA/AA & $1.32(0.80,2.17)$ & 0.276 & $0.8 \mathrm{I}(0.42, I .55)$ & 0.517 \\
\hline Additive model & $1.20(0.88,1.63)$ & 0.249 & $0.89(0.60,1.36)$ & 0.581 \\
\hline \multicolumn{5}{|l|}{ rsII43634 } \\
\hline GG & Reference & & Reference & \\
\hline GA & $0.60(0.19,1.86)$ & 0.371 & I.43(0.33,6.27) & 0.634 \\
\hline AA & - & - & - & - \\
\hline GA/AA & $0.70(0.24,2.03)$ & 0.506 & I.43(0.33,6.27) & 0.634 \\
\hline Additive model & $0.82(0.32,2.10)$ & 0.687 & I.43(0.33,6.27) & 0.634 \\
\hline \multicolumn{5}{|l|}{ ILIA } \\
\hline \multicolumn{5}{|l|}{ rs|800587 } \\
\hline GG & Reference & & Reference & \\
\hline GA & $1.20(0.68,2.14)$ & 0.531 & $1.50(0.68,3.33)$ & 0.317 \\
\hline AA & $1.40(0.08,24.45)$ & 0.819 & $0.60(0.08,4.49)$ & 0.622 \\
\hline GA/AA & $1.21(0.68,2.13)$ & 0.515 & I.35(0.64,2.88) & 0.432 \\
\hline Additive model & $1.20(0.70,2.06)$ & 0.512 & I.18(0.62,2.27) & 0.615 \\
\hline \multicolumn{5}{|l|}{ PTGS2 } \\
\hline \multicolumn{5}{|l|}{ rs204I7 } \\
\hline $\mathrm{CC}$ & Reference & & Reference & \\
\hline GC & $0.89(0.41,1.92)$ & 0.769 & I.I I $(0.48,2.52)$ & 0.804 \\
\hline GG & - & - & - & - \\
\hline GC/GG & $0.89(0.41,1.92)$ & 0.769 & I.I I $(0.49,2.52)$ & 0.804 \\
\hline Additive model & $0.89(0.41,1.92)$ & 0.769 & I. $10(0.49,2.52)$ & 0.804 \\
\hline \multicolumn{5}{|l|}{ rs689466 } \\
\hline $\mathrm{TT}$ & Reference & & Reference & \\
\hline CT & $0.73(0.45, I .18)$ & 0.197 & $0.8 \mathrm{I}(0.44, \mathrm{I} .47)$ & 0.483 \\
\hline $\mathrm{CC}$ & $0.47(0.11,2.08)$ & 0.325 & $0.46(0.11,1.95)$ & 0.293 \\
\hline $\mathrm{CT} / \mathrm{CC}$ & $0.7 I(0.44, I . \mid 3)$ & 0.151 & $0.76(0.42,1.36)$ & 0.357 \\
\hline Additive model & $0.72(0.48, I .10)$ & 0.130 & $0.75(0.46,1.24)$ & 0.266 \\
\hline \multicolumn{5}{|l|}{ rs5275 } \\
\hline AA & Reference & & Reference & \\
\hline GA & $0.77(0.46, I .28)$ & 0.310 & I.24(0.64,2.4I) & 0.517 \\
\hline GG & I.77(0.93,3.39) & 0.085 & I.16(0.48,2.79) & 0.744 \\
\hline GA/GG & $0.97(0.61,1.55)$ & 0.899 & $\mid .21(0.65,2.27)$ & 0.552 \\
\hline Additive model & $1.20(0.88,1.64)$ & 0.254 & I.08(0.7I,I.64) & 0.713 \\
\hline
\end{tabular}


Table 2 (Continued).

\begin{tabular}{|c|c|c|c|c|}
\hline \multirow[t]{2}{*}{ Genotype } & \multicolumn{2}{|c|}{ Male } & \multicolumn{2}{|c|}{ Female } \\
\hline & $\operatorname{OR}(95 \% \mathrm{CI})^{\mathrm{a}}$ & P-value ${ }^{a}$ & $\operatorname{OR}(95 \% \mathrm{CI})^{\mathrm{a}}$ & P-value ${ }^{a}$ \\
\hline \multicolumn{5}{|l|}{ MMP9 } \\
\hline \multicolumn{5}{|l|}{ rsI7576 } \\
\hline GG & Reference & & Reference & \\
\hline GA & $0.93(0.58,1.49)$ & 0.768 & $1.23(0.66,2.30)$ & 0.518 \\
\hline$A A$ & $1.01(0.48,2.12)$ & 0.987 & I.I7(0.47,2.90) & 0.742 \\
\hline GA/AA & $0.95(0.61,1.47)$ & $0.80 \mathrm{I}$ & I.12(0.68,2.16) & 0.513 \\
\hline Additive model & $0.97(0.70-1.36)$ & 0.889 & $1.12(0.74-1.70)$ & 0.592 \\
\hline \multicolumn{5}{|l|}{ rs39|8242 } \\
\hline $\mathrm{CC}$ & Reference & & Reference & \\
\hline $\mathrm{CT}$ & I.I $3(0.62,2.04)$ & 0.689 & $1.96(0.82,4.69)$ & 0.132 \\
\hline TT & $2.52(0.35,18.32)$ & 0.363 & - & - \\
\hline $\mathrm{CT} / \mathrm{TT}$ & $1.19(0.67,2.12)$ & 0.552 & $1.53(0.67,3.48)$ & 0.315 \\
\hline Additive model & $1.23(0.73-2.06)$ & 0.442 & $1.18(0.56-2.48)$ & 0.665 \\
\hline \multicolumn{5}{|l|}{ rs9509 } \\
\hline TT & Reference & & Reference & \\
\hline $\mathrm{CT}$ & $0.96(0.60,1.54)$ & 0.867 & $0.76(0.4 I, I .4 I)$ & 0.389 \\
\hline $\mathrm{CC}$ & $0.70(0.23,2.14)$ & 0.531 & $0.47(0.10,2.25)$ & 0.346 \\
\hline $\mathrm{CT} / \mathrm{CC}$ & $0.93(0.59-1.46)$ & 0.742 & $0.73(0.40-1.32)$ & 0.295 \\
\hline Additive model & $0.91(0.62,1.34)$ & $0.64 I$ & $0.73(0.44, I .23)$ & 0.236 \\
\hline \multicolumn{5}{|l|}{ MMP2 } \\
\hline \multicolumn{5}{|l|}{ rs720I } \\
\hline$A A$ & Reference & & Reference & \\
\hline $\mathrm{CA}$ & $1.08(0.67,1.74)$ & 0.757 & $0.79(0.42, I .50)$ & 0.471 \\
\hline $\mathrm{CC}$ & I.I8(0.48,2.90) & 0.713 & I. $10(0.30,4.05)$ & 0.890 \\
\hline $\mathrm{CA} / \mathrm{CC}$ & I.IO(0.70,I.73) & 0.683 & $0.86(0.47, I .55)$ & 0.611 \\
\hline Additive model & $1.09(0.77-1.55)$ & 0.635 & $0.93(0.57-1.53)$ & 0.785 \\
\hline \multicolumn{5}{|l|}{ rs2285053 } \\
\hline $\mathrm{CC}$ & Reference & & Reference & \\
\hline $\mathrm{CT}$ & $0.8 I(0.5 I, I .3 I)$ & 0.394 & I. $40(0.74,2.63)$ & 0.304 \\
\hline TT & $1.44(0.62,3.32)$ & 0.394 & I.I5(0.39,3.46) & 0.797 \\
\hline $\mathrm{CT} / \mathrm{TT}$ & $0.90(0.58, I .4 I)$ & 0.650 & I.34(0.74,2.43) & 0.327 \\
\hline Additive model & I.0I(0.72-I.44) & 0.917 & $1.20(0.76-1.90)$ & 0.438 \\
\hline \multicolumn{5}{|l|}{ rs243864 } \\
\hline TT & Reference & & Reference & \\
\hline $\mathrm{CT}$ & $0.84(0.48-1.49)$ & 0.550 & $1.16(0.55-2.45)$ & 0.699 \\
\hline $\mathrm{CC}$ & $9.40(0.88-100.44)$ & 0.064 & $0.89(0.13-6.14)$ & 0.909 \\
\hline $\mathrm{CT} / \mathrm{CC}$ & $0.96(0.56-1.68)$ & 0.897 & I.II(0.55-2.26) & 0.770 \\
\hline Additive model & $1.10(0.66-1.84)$ & 0.705 & $1.05(0.58-1.92)$ & 0.864 \\
\hline
\end{tabular}

Note: ${ }^{a}$ Adjusted for age, sex, smoking, and drinking.

of IS, no statistically significant association was observed; thus, large-sample studies should be confirmed in the Chinese population. Admittedly, there were some limitations of this study, such as, the genes enrolled in this study may be affected by many SNPs, here we selected some of them, therefore our study was not comprehensive enough; moreover, the study failed to assess the protein expression in patients, which protein plays a decisive role; finally, the sample size of this study was relatively small, which may affect the statistical power. 
Table 3 Association Between Genetic Variations and Types of Ischemic Stroke Risk

\begin{tabular}{|c|c|c|c|c|c|c|c|c|}
\hline \multirow[t]{2}{*}{ Genotype } & \multicolumn{2}{|c|}{ Small-Vessel Occlusion } & \multicolumn{2}{|l|}{ Large-Artery } & \multicolumn{2}{|l|}{ Cardioembolism } & \multicolumn{2}{|l|}{ Other Type } \\
\hline & OR $(95 \% \mathrm{CI})^{\mathrm{a}}$ & $P$ value & OR $(95 \% \mathrm{Cl})^{\mathrm{a}}$ & $P$ value & OR $(95 \% \mathrm{CI})^{a}$ & $P$ value & OR $(95 \% \mathrm{CI})^{\mathrm{a}}$ & $P$ value \\
\hline \multicolumn{9}{|l|}{ rs| 800587} \\
\hline GG & Reference & & Reference & & Reference & & Reference & \\
\hline GA & $0.59(0.29-1.21)$ & 0.151 & $0.93(0.50-1.74)$ & 0.828 & $0.89(0.31-2.57)$ & 0.822 & $0.76(0.40-1.44)$ & 0.399 \\
\hline AA & - & - & $0.65(0.09-4.50)$ & 0.658 & - & - & $0.63(0.09-4.26)$ & 0.632 \\
\hline GA/AA & $0.63(0.31-1.28)$ & 0.202 & $0.91(0.50-1.66)$ & 0.751 & $0.97(0.34-2.78)$ & 0.958 & $0.75(0.4 \mid-1.38)$ & 0.354 \\
\hline Additive model & $0.70(0.36-1.37)$ & 0.299 & $0.89(0.53-1.54)$ & 0.694 & $1.06(0.40-2.82)$ & 0.906 & $0.77(0.45-1.33)$ & 0.346 \\
\hline \multicolumn{9}{|l|}{ rs689466 } \\
\hline TT & Reference & & Reference & & Reference & & Reference & \\
\hline CT & $2.51(1.22-5.16)$ & 0.012 & $0.86(0.54-1.38)$ & 0.535 & $1.45(0.58-3.63)$ & 0.432 & $1.49(0.88-2.53)$ & 0.138 \\
\hline $\mathrm{CC}$ & $3.27(0.40-26.84)$ & 0.270 & $1.83(0.37-9.08)$ & 0.461 & $0.91(0.15-5.39)$ & 0.914 & $2.33(0.49-11.16)$ & 0.290 \\
\hline СT/CC & $2.53(1.26-5.07)$ & 0.009 & $0.91(0.57-1.45)$ & 0.686 & $1.35(0.57-3.23)$ & 0.499 & $1.56(0.93-2.61)$ & 0.094 \\
\hline Additive model & $2.26(1.19-4.28)$ & 0.013 & $0.99(0.65-1.49)$ & 0.941 & $1.18(0.58-2.45)$ & 0.641 & $1.51(0.96-2.40)$ & 0.078 \\
\hline \multicolumn{9}{|l|}{ rs3918242 } \\
\hline $\mathrm{CC}$ & Reference & & Reference & & Reference & & Reference & \\
\hline CT & $1.25(0.49-3.19)$ & $0.64 I$ & $0.87(0.46-1.61)$ & 0.648 & $0.31(0.1 I-0.91)$ & 0.033 & $0.62(0.33-1.17)$ & 0.137 \\
\hline $\mathrm{TT}$ & $0.27(0.05-1.58)$ & 0.146 & $2.23(0.29-25.14)$ & 0.517 & - & - & - & - \\
\hline $\mathrm{CT} / \mathrm{TT}$ & $1.01(0.44-2.32)$ & 0.987 & $0.92(0.50-1.69)$ & 0.790 & $0.39(0.14-1.09)$ & 0.072 & $0.69(0.37-1.29)$ & 0.239 \\
\hline Additive model & $0.86(0.43-1.71)$ & 0.661 & $0.98(0.57-1.71)$ & 0.954 & $0.55(0.23-1.35)$ & 0.192 & $0.80(0.45-0.42)$ & 0.435 \\
\hline \multicolumn{9}{|l|}{ rs5275 } \\
\hline $\mathrm{AA}$ & Reference & & Reference & & Reference & & Reference & \\
\hline GA & $0.49(0.22-1.07)$ & 0.072 & $1.28(0.76-2.15)$ & 0.350 & $1.47(0.55-3.97)$ & 0.443 & $1.18(0.67-2.07)$ & 0.574 \\
\hline GG & $0.31(0.12-0.80)$ & 0.016 & $0.97(0.50-1.88)$ & 0.927 & $0.83(0.24-2.95)$ & 0.775 & $0.54(0.27-1.10)$ & 0.089 \\
\hline GA/GG & $0.45(0.21-0.95)$ & 0.036 & $1.20(0.74-1.93)$ & 0.462 & $1.20(0.49-2.96)$ & 0.686 & $0.96(0.57,1.61)$ & 0.874 \\
\hline Additive model & $0.60(0.39-0.92)$ & 0.020 & $1.05(0.75-1.46)$ & 0.782 & $0.94(0.50-1.78)$ & 0.855 & $0.8 I(0.57,1.14)$ & 0.217 \\
\hline
\end{tabular}

Note: ${ }^{a}$ Adjusted for age, sex, smoking, and drinking; the results with a significant difference are in bold.

In summary, our study suggested that $P T G S 2$ rs689466 $\mathrm{C}$ and rs5275 A were potentially associated with IS subtype of small-vessel occlusion risk. Our result should be confirmed with further large sample sized studies.

\section{Ethical Statement and Consent}

The study complied with the Declaration of Helsinki and was approved by the Institutional Review Board of the Nanjing First Hospital, and all participants were written informed consent.

\section{Author Contributions}

All authors contributed to data analysis, drafting or revising the article, have agreed on the journal to which the article will be submitted, gave final approval of the version to be published, and agree to be accountable for all aspects of the work.

\section{Funding}

This study was supported by the Innovation Team of Jiangsu Provincial Health-Strengthening Engineering by
Science and Education (CXTDB2017008) and Jiangsu Youth Medical Talents Training Project to $\mathrm{BH}$ (QNRC2016066).

\section{Disclosure}

The authors report no conflicts of interest in this work.

\section{References}

1. Diseases GBD, Injuries C. Global burden of 369 diseases and injuries in 204 countries and territories, 1990-2019: a systematic analysis for the Global Burden of Disease Study 2019. Lancet. 2020;396 (10258):1204-1222.

2. Adams HP Jr., Bendixen BH, Kappelle LJ, et al. Classification of subtype of acute ischemic stroke. Definitions for use in a multicenter clinical trial. TOAST. Trial of Org 10172 in Acute Stroke Treatment. Stroke. 1993;24(1):35-41. doi:10.1161/01.STR.24.1.35

3. Zhang LF, Yang J, Hong Z, et al. Proportion of different subtypes of stroke in China. Stroke. 2003;34(9):2091-2096. doi:10.1161/01. STR.0000087149.42294.8C

4. Bevan S, Traylor M, Adib-Samii P, et al. Genetic heritability of ischemic stroke and the contribution of previously reported candidate gene and genomewide associations. Stroke. 2012;43(12):3161-3167. doi:10.1161/STROKEAHA.112.665760 
5. Kelly PJ, Lemmens R, Tsivgoulis G. Inflammation and stroke risk: a new target for prevention. Stroke. 2021;52(8):2697-2706. doi:10.1161/STROKEAHA.121.034388.

6. Della Corte V, Tuttolomondo A, Pecoraro R, Di Raimondo D, Vassallo V, Pinto A. Inflammation, endothelial dysfunction and arterial stiffness as therapeutic targets in cardiovascular medicine. Curr Pharm Des. 2016;22 (30):4658-4668. doi:10.2174/1381612822666160510124801

7. Pei J, You X, Fu Q. Inflammation in the pathogenesis of ischemic stroke. Front Biosci. 2015;20(4):772-783. doi:10.2741/4336

8. Shekhar S, Cunningham MW, Pabbidi MR, Wang S, Booz GW, Fan F. Targeting vascular inflammation in ischemic stroke: recent developments on novel immunomodulatory approaches. Eur J Pharmacol. 2018;833:531-544. doi:10.1016/j.ejphar.2018.06.028

9. Zare Rafie M, Esmaeilzadeh A, Ghoreishi A, Tahmasebi S, Faghihzadeh E, Elahi R. IL-38 as an early predictor of the ischemic stroke prognosis. Cytokine. 2021;146:155626. doi:10.1016/j. cyto. 2021.155626

10. Lehmann A, Alfieri DF, de Araujo MCM, et al. Immuneinflammatory, coagulation, adhesion, and imaging biomarkers combined in machine learning models improve the prediction of death 1 year after ischemic stroke. Clin Exp Med. 2021:1-3. doi:10.1007/ s10238-021-00732-w.

11. Palm F, Aigner A, Pussinen PJ, et al. Association of a multigenetic pro-inflammatory profile with ischaemic stroke. Cerebrovasc Dis. 2020;49(2):170-176. doi:10.1159/000507042

12. Dinarello CA, Simon A, van der Meer JW. Treating inflammation by blocking interleukin-1 in a broad spectrum of diseases. Nat Rev Drug Discov. 2012;11(8):633-652.

13. Goracy I, Kaczmarczyk M, Ciechanowicz A, et al. Polymorphism of interleukin 1B may modulate the risk of ischemic stroke in polish patients. Medicine. 2019;55(9):558.

14. Tuttolomondo A, Pecoraro R, Casuccio A, et al. Peripheral frequency of CD4+ CD28- cells in acute ischemic stroke: relationship with stroke subtype and severity markers. Medicine. 2015;94(20):e813. doi:10.1097/MD.0000000000000813

15. Li WX, Dai SX, Wang Q, et al. Integrated analysis of ischemic stroke datasets revealed sex and age difference in anti-stroke targets. PeerJ. 2016;4:e2470. doi:10.7717/peerj.2470

16. Hellmann J, Tang Y, Zhang MJ, et al. Atf3 negatively regulates Ptgs2/ Cox2 expression during acute inflammation. Prostaglandins Other Lipid Mediat. 2015;116-117:49-56. doi:10.1016/j.prostaglandins.2015.01.001

17. $\mathrm{Su} \mathrm{Z}, \mathrm{Wu} \mathrm{F}$. Inflammatory factors induce thrombosis through the miR-146b-3p/p38MAPK/COX-2 pathway. Biomed Res Int 2020;2020:8718321. doi:10.1155/2020/8718321

18. Wu G, Cai H, Cai H, et al. Influence of the cyclooxygenase-2 gene $-765 \mathrm{G} / \mathrm{C}$ and $-1195 \mathrm{G} / \mathrm{A}$ polymorphisms on development of ischemic stroke. J Stroke Cerebrovasc Dis. 2016;25(9):2126-2135. doi:10.1016/j.jstrokecerebrovasdis.2016.06.001

19. Oliveira-Filho J, Ornellas ACP, Zhang CR, et al. COX-2 rs20417 polymorphism is associated with stroke and white matter disease. J Stroke Cerebrovasc Dis. 2015;24(8):1817-1822. doi:10.1016/j. jstrokecerebrovasdis.2015.04.018

20. Galis ZS, Khatri JJ. Matrix metalloproteinases in vascular remodeling and atherogenesis: the good, the bad, and the ugly. Circ Res. 2002;90 (3):251-262. doi:10.1161/res.90.3.251

21. Anthony DC, Ferguson B, Matyzak MK, Miller KM, Esiri MM, Perry VH. Differential matrix metalloproteinase expression in cases of multiple sclerosis and stroke. Neuropathol Appl Neurobiol. 1997;23(5):406-415. doi:10.1111/j.1365-2990.1997.tb01315.x

22. Clark AW, Krekoski CA, Bou SS, Chapman KR, Edwards DR. Increased gelatinase A (MMP-2) and gelatinase B (MMP-9) activities in human brain after focal ischemia. Neurosci Lett. 1997;238(12):53-56. doi:10.1016/S0304-3940(97)00859-8
23. Lo EH, Wang X, Cuzner ML. Extracellular proteolysis in brain injury and inflammation: role for plasminogen activators and matrix metalloproteinases. J Neurosci Res. 2002;69(1):1-9. doi:10.1002/ jnr. 10270

24. Chang JJ, Stanfill A, Pourmotabbed T. The role of matrix metalloproteinase polymorphisms in ischemic stroke. Int J Mol Sci. 2016;17 (8):1323. doi:10.3390/ijms17081323

25. Wang L, Deng L, Yuan R, Liu J, Li Y, Liu M. Association of matrix metalloproteinase 9 and cellular fibronectin and outcome in acute ischemic stroke: a systematic review and meta-analysis. Front Neurol. 2020;11:523506. doi:10.3389/fneur.2020.523506

26. Rempe RG, Hartz AMS, Bauer B. Matrix metalloproteinases in the brain and blood-brain barrier: versatile breakers and makers. J Cereb Blood Flow Metab. 2016;36(9):1481-1507. doi:10.1177/0271678X16655551

27. Zhong C, Yang J, Xu T, et al. Serum matrix metalloproteinase-9 levels and prognosis of acute ischemic stroke. Neurology. 2017;89 (8):805-812. doi:10.1212/WNL.0000000000004257

28. Niu F, Wei B, Yan M, Li J, Ouyang Y, Jin T. Matrix metalloproteinase-2 gene polymorphisms are associated with ischemic stroke in a Hainan population. Medicine. 2018;97(39): e12302. doi:10.1097/MD.0000000000012302

29. Guo D, Zhu Z, Zhong C, et al. Prognostic metrics associated with inflammation and atherosclerosis signaling evaluate the burden of adverse clinical outcomes in ischemic stroke patients. Clin Chem. 2020;66(11):1434-1443. doi:10.1093/clinchem/hvaa201

30. Pan Y, He B, Sun H, et al. Susceptibility of PON1/PON2 genetic variations to ischemic stroke risk in a Chinese Han population. Pharmgenomics Pers Med. 2020;13:563-570.

31. Vargas-Alarcon G, Perez-Mendez O, Gonzalez-Pacheco H, et al. The rs508487, rs236911, and rs236918 genetic variants of the proprotein convertase subtilisin-kexin type 7 (PCSK7) gene are associated with acute coronary syndrome and with plasma concentrations of HDL-cholesterol and triglycerides. Cells. 2021;10(6):1444. doi:10.3390/cells10061444

32. Revilla M, Obach V, Cervera A, Davalos A, Castillo J, Chamorro A. A $-174 \mathrm{G} / \mathrm{C}$ polymorphism of the interleukin- 6 gene in patients with lacunar infarction. Neurosci Lett. 2002;324(1):29-32. doi:10.1016/ S0304-3940(02)00169-6

33. Lindsberg PJ, Grau AJ. Inflammation and infections as risk factors for ischemic stroke. Stroke. 2003;34(10):2518-2532. doi:10.1161/01. STR.0000089015.51603.CC

34. Tuttolomondo A, Maida C, Pinto A. Diabetic foot syndrome as a possible cardiovascular marker in diabetic patients. $J$ Diabetes Res. 2015;2015:268390. doi:10.1155/2015/268390

35. Tuttolomondo A, Di Raimondo D, Pecoraro R, et al. HLA and killer cell immunoglobulin-like receptor (KIRs) genotyping in patients with acute ischemic stroke. $J$ Neuroinflammation. 2019;16(1):88. doi:10.1186/s12974-019-1469-5

36. Herschman HR. Function and regulation of prostaglandin synthase 2 . Adv Exp Med Biol. 1999;469:3-8.

37. Chen GZ, Shan XY, Cheng GP, Tao HM. Cyclooxygenase-2 genetic polymorphism and stroke subtypes in Chinese. J Mol Neurosci. 2013;51(2):467-473. doi:10.1007/s12031-013-0078-5

38. Li J, Lu X, Zou X, et al. COX-2 rs5275 and rs689466 polymorphism and risk of lung cancer: a PRISMA-compliant meta-analysis. Medicine. 2018;97(35):e11859. doi:10.1097/MD.0000000000011859

39. Lurie G, Terry KL, Wilkens LR, et al. Pooled analysis of the association of PTGS2 rs5275 polymorphism and NSAID use with invasive ovarian carcinoma risk. Cancer Causes Control. 2010;21 (10):1731-1741. doi:10.1007/s10552-010-9602-x

40. Shan XY, Chen GZ, Cheng GP, Tao HM. Cyclooxygenase 2 genetic polymorphism may increase the risk of developing leukoaraiosis in Chinese. J Mol Neurosci. 2013;51(2):461-466. doi:10.1007/s12031013-0066-9 
41. Zhao L, Fang J, Zhou M, et al. Interaction between COX-1 and COX-2 increases susceptibility to ischemic stroke in a Chinese population. BMC Neurol. 2019;19(1):291. doi:10.1186/s12883-0191505-1

42. Maguire J, Thakkinstian A, Levi C, et al. Impact of COX-2 rs5275 and rs20417 and GPIIIa rs5918 polymorphisms on 90-day ischemic stroke functional outcome: a novel finding. J Stroke Cerebrovasc Dis. 2011;20(2):134-144. doi:10.1016/j.jstrokecerebrovasdis.2009.10.011

43. Romanic AM, White RF, Arleth AJ, Ohlstein EH, Barone FC. Matrix metalloproteinase expression increases after cerebral focal ischemia in rats: inhibition of matrix metalloproteinase- 9 reduces infarct size. Stroke. 1998;29(5):1020-1030. doi:10.1161/01.STR.29.5.1020

44. Zhang B, Ye S, Herrmann SM, et al. Functional polymorphism in the regulatory region of gelatinase $\mathrm{B}$ gene in relation to severity of coronary atherosclerosis. Circulation. 1999;99(14):1788-1794. doi:10.1161/01.CIR.99.14.1788

45. Ye S. Polymorphism in matrix metalloproteinase gene promoters: implication in regulation of gene expression and susceptibility of various diseases. Matrix Biol. 2000;19(7):623-629. doi:10.1016/ S0945-053X(00)00102-5
46. Zhang B, Henney A, Eriksson P, Hamsten A, Watkins H, Ye S. Genetic variation at the matrix metalloproteinase-9 locus on chromosome 20q12.2-13.1. Hum Genet. 1999;105(5):418-423.doi:10.1007/ s004390051124.

47. Montaner J, Fernandez-Cadenas I, Molina CA, et al. Safety profile of tissue plasminogen activator treatment among stroke patients carrying a common polymorphism $(\mathrm{C}-1562 \mathrm{~T})$ in the promoter region of the matrix metalloproteinase-9 gene. Stroke. 2003;34(12):2851-2855. doi:10.1161/01.STR.0000098648.54429.1C

48. Zhao JH, Xu YM, Xing HX, et al. Associations between matrix metalloproteinase gene polymorphisms and the development of cerebral infarction. Gene Mol Res. 2016;14(4):19418-19424. doi:10.4238/2015.December.30.3

49. He T, Wang J, Wang XL, Deng WS, Sun P. Association between the matrix metalloproteinase-9 rs3918242 polymorphism and ischemic stroke susceptibility: a meta-analysis. J Stroke Cerebrovasc Dis. 2017;26 (5):1136-1143. doi:10.1016/j.jstrokecerebrovasdis.2016.12.036

\section{Publish your work in this journal}

Pharmacogenomics and Personalized Medicine is an international, peer-reviewed, open access journal characterizing the influence of genotype on pharmacology leading to the development of personalized treatment programs and individualized drug selection for improved safety, efficacy and sustainability. This journal is indexed on the American Chemical Society's Chemical Abstracts Service (CAS). The manuscript management system is completely online and includes a very quick and fair peer-review system, which is all easy to use. Visit http://www.dovepress.com/testimonials.php to read real quotes from published authors. 